TRANSACTIONS OF THE

AMERICAN MATHEMATICAL SOCIETY

Volume 354, Number 4, Pages 1453-1471

S 0002-9947(01)02792-1

Article electronically published on November 21, 2001

\title{
SRB MEASURES AND PESIN'S ENTROPY FORMULA FOR ENDOMORPHISMS
}

\author{
MIN QIAN AND SHU ZHU
}

\begin{abstract}
We present a formulation of the SRB (Sinai-Ruelle-Bowen) property for invariant measures of $C^{2}$ endomorphisms (maybe non-invertible and with singularities) of a compact manifold via their inverse limit spaces, and prove that this property is necessary and sufficient for Pesin's entropy formula. This result is a non-invertible endomorphisms version of a result of Ledrappier, Strelcyn and Young.
\end{abstract}

\section{INTRODUCTION}

Pesin's entropy formula relates two important concepts, Lyapunov exponents and measure-theoretic entropy, and it plays an important role in the smooth ergodic theory of dynamical systems. There are extensive results concerning Pesin's entropy formula in both deterministic dynamical systems and random dynamical systems of diffeomorphisms. In [11], Pesin showed that if an invariant measure of a $C^{2}$ diffeomorphism of a compact manifold is absolutely continuous with respect to the Lebesgue measure of the manifold, then it satisfies Pesin's identity. (See 9] for an alternative proof.) Then it was conjectured by Ruelle, and proved by Ledrappier, Strelcyn and Young in [4] and [5], that an invariant measure of a $C^{2}$ diffeomorphism satisfies Pesin's entropy formula if and only if it has absolutely continuous conditional measures on unstable manifolds. The latter property is known as the SRB property of the corresponding invariant measure of the diffeomorphism. The above results were successfully generalized to random dynamical systems of diffeomorphisms [6, 8, 1]. In the literature, there are also considerable results concerning non-invertible systems, such as for expanding maps [2, 10, Axiom A endomorphisms [13], and smooth measures of $C^{2}$ endomorphisms [7, 17]-Pesin's entropy formula also hold. However, the necessary and sufficient condition for Pesin's entropy formula in the case of endomorphisms is still lacking.

For an invariant measure of a diffeomorphism, the SRB property is understood as that it induces absolutely continuous conditional measures on unstable manifolds. In general this definition cannot be used for endomorphisms, since each unstable manifold usually depends on the whole orbit and no foliation structure is available for unstable manifolds. In this paper, we present a formulation of the SRB property for invariant measures of $C^{2}$ endomorphisms of a compact smooth manifold via their

Received by the editors January 27, 1999 and, in revised form, December 21, 1999.

1991 Mathematics Subject Classification. Primary 58F11; Secondary 28D05, 28D20.

Key words and phrases. Entropy, Lyapunov exponent, SRB measure.

This research is supported by the National Natural Science Foundation of China.

The first author supported by the Special Funds for Major State Basic Research Projects.

(C)2001 American Mathematical Society 
inverse limit spaces, and then prove that this property is necessary and sufficient for Pesin's entropy formula. This is a non-invertible version of the main result of Ledrappier, Strelcyn and Young [4, 5]. An interesting consequence of the main theorem is that an invariant measure of a $C^{2}$ endomorphism has this SRB property if it is absolutely continuous with respect to the Lebesgue measure of the manifold. Invariant measures having this SRB property also exist on Axiom A attractors of $C^{2}$ endomorphisms.

As compared with the case of diffeomorphisms, three main difficulties arise while dealing with endomorphisms. The first is the formulation of the SRB property. The second comes from singularities. However, a natural integrability condition (Condition (1.6) ) will enable us to eliminate this difficulty easily. The final difficulty arises from the non-invertibility. To overcome this deficiency, the inverse limit space has to be introduced. At first impression, the deterministic non-invertible dynamical system is quite different from random diffeomorphisms. However, one will see from this paper that when the inverse limit space is introduced, the non-invertible case is very close to the case of random diffeomorphisms, for which a systematic treatment is now available in [8]. Therefore, with some necessary modifications, many ideas and techniques used in [8] can be applied to our present study. This is the first example showing that a deterministic problem can be successfully solved by employing techniques developed for random dynamical systems.

The paper is organized as follows. In Section 1 we present our formulation of the SRB property and state the main result of the paper. In Section 2, we give some preliminary results. Section 3 consists of some technical preparations for the proof of Theorem 1.1 The proof of the sufficientcy part of the theorem will be completed in Section 4, and we present the key points for the proof of necessity in Section 5.

\section{Formulation and Main Result}

Throughout this paper, $M$ will always be a $C^{\infty}, m_{0}$-dimensional and compact Riemannian manifold without boundary. Let $f: M \rightarrow M$ be a $C^{2}$ map. The inverse limit space of $f$, written $M^{f}$, is a closed subset of $M^{\mathbb{Z}}$ (endowed with the product topology and the metric $d(\tilde{x}, \tilde{y})=\sum_{i=-\infty}^{+\infty} 2^{-|i|} d\left(x_{i}, y_{i}\right)$ for $\tilde{x}=\left\{x_{i}\right\}_{i \in \mathbb{Z}}$, $\left.\tilde{y}=\left\{y_{i}\right\}_{i \in \mathbb{Z}} \in M^{\mathbb{Z}}\right)$ consisting of all full orbits of $f$, i.e.,

$$
M^{f}=\left\{\tilde{x}=\left\{x_{i}\right\}_{i \in \mathbb{Z}} \mid x_{i} \in M, f\left(x_{i}\right)=x_{i+1}, \forall i \in \mathbb{Z}\right\}
$$

Let $\pi$ be the natural projection from $M^{f}$ to $M$, i.e.,

$$
\pi(\tilde{x})=x_{0}, \quad \forall \tilde{x} \in M^{f},
$$

and let $\tau: M^{f} \rightarrow M^{f}$ denote the left shift homeomorphism. Clearly, $\pi \circ \tau=f \circ \pi$.

In what follows we always assume that $\mu$ is an $f$-invariant Borel probability measure. According to Rokhlin [14, there is a unique $\tau$-invariant Borel probability measure $\tilde{\mu}$ on $M^{f}$ which is projected to $\mu$ by $\pi$. Let

$$
+\infty>\lambda^{(1)}(x)>\lambda^{(2)}(x)>\cdots>\lambda^{(s(x))}(x) \geq-\infty
$$

be the Lyapunov exponents of $f$ at $x$ and let $m_{i}(x)$ be the multiplicity of $\lambda^{(i)}(x)$.

Let $\tilde{x} \in M^{f}$ and let $W_{l o c}^{u}(\tilde{x})$ be a $k(\tilde{x})$-dimensional $C^{1,1}$ embedded submanifold of $M$, where $k(\tilde{x})=\sum_{\lambda^{(i)}\left(x_{0}\right)>0} m_{i}\left(x_{0}\right)$. We call $W_{l o c}^{u}(\tilde{x})$ a local unstable manifold 
of $f$ at $\tilde{x}$ if there are numbers $\lambda>0,0<\varepsilon<\lambda / 200,0<C_{1} \leq 1 \leq C_{2}$, and for each $y_{0} \in W_{l o c}^{u}(\tilde{x})$ there exists a unique $\tilde{y}=\left\{y_{n}\right\}_{n \in \mathbb{Z}} \in M^{f}$ such that $\pi \tilde{y}=y_{0}$,

$$
d\left(y_{-n}, x_{-n}\right) \leq C_{1} e^{-n \varepsilon}, \quad \forall n \in \mathbb{N},
$$

and

$$
d\left(y_{-n}, x_{-n}\right) \leq C_{2} e^{-n \lambda}, \quad \forall n \in \mathbb{N} .
$$

We will write

$$
\widetilde{W}_{l o c}^{u}(\tilde{x})=\left\{\tilde{y} \in M^{f} \mid y_{0} \in W_{l o c}^{u}(\tilde{x}), \tilde{y} \text { satisfies (1.1) and (1.2) }\right\} .
$$

The global unstable manifold of $f$ at $\tilde{x}$ is defined as

$$
W^{u}(\tilde{x})=\left\{y_{0} \in M \mid \exists \tilde{y} \in M^{f} \text { with } \pi \tilde{y}=y_{0} \text { and } \varlimsup_{n \rightarrow+\infty} \frac{1}{n} \log d\left(x_{-n}, y_{-n}\right)<0\right\}
$$

and we will write

$$
\widetilde{W}^{u}(\tilde{x})=\left\{\tilde{y} \in M^{f} \mid \varlimsup_{n \rightarrow+\infty} \frac{1}{n} \log d\left(x_{-n}, y_{-n}\right)<0\right\} .
$$

We will always assume moreover that the system $f:(M, \mu) \rightarrow(M, \mu)$ satisfies the integrability condition

$$
\log \left|\operatorname{det} T_{x} f\right| \in L^{1}(M, \mu) .
$$

Then it can be shown that for $\tilde{\mu}$-a.e. $\tilde{x} \in M^{f}$, if $\lambda^{(1)}\left(x_{0}\right)>0$, then the local unstable manifold $W_{l o c}^{u}(\tilde{x})$ exists and there exists a sequence of $C^{1,1}$ embedded $k(\tilde{x})$-dimensional discs $\left\{W_{-n}(\tilde{x})\right\}_{n=0}^{+\infty}$ in $M$ such that $f W_{-n}(\tilde{x}) \supset W_{-n+1}(\tilde{x})$ for all $n \geq 1$,

$$
W^{u}(\tilde{x})=\bigcup_{n=0}^{+\infty} f^{n} W_{-n}(\tilde{x})
$$

and, if $\lambda^{(i)}\left(x_{0}\right) \leq 0$ for all $1 \leq i \leq s\left(x_{0}\right)$, then

$$
W^{u}(\tilde{x})=\left\{x_{0}\right\} .
$$

See [19] for a detailed treatment of this topic.

Definition 1.1. A measurable partition $\eta$ of $M^{f}$ is said to be subordinate to $W^{u}$ manifolds of $(f, \mu)$ if for $\tilde{\mu}$-a.e. $\tilde{x}, \eta(\tilde{x})$ has the following properties:

(1) $\left.\pi\right|_{\eta(\tilde{x})}: \eta(\tilde{x}) \rightarrow \pi(\eta(\tilde{x}))$ is bijective.

(2) There exists a $k(\tilde{x})$-dimensional $C^{1,1}$ embedded submanifold $W_{\tilde{x}}$ of $M$ such that $W_{\tilde{x}} \subset W^{u}(\tilde{x})$,

$$
\pi(\eta(\tilde{x})) \subset W_{\tilde{x}}
$$

and $\pi(\eta(\tilde{x}))$ contains an open neighborhood of $x_{0}$ in $W_{\tilde{x}}$, this neighborhood being taken in the topology of $W_{\tilde{x}}$ as a submanifold (or a subset) of $M$.

In Section 3, we will show that there are always measurable partitions subordinate to $W^{u}$-manifolds of $(f, \mu)$.

We are now prepared to give the definition of the SRB property for $f$-invariant Borel probability measures. We first give a somewhat intuitive description of this property. Let $f:(M, \mu) \rightarrow(M, \mu)$ be as given above. Let $\widetilde{V}$ be a Borel subset of $M^{f}$ with $\tilde{\mu}(\widetilde{V})>0$. Suppose that $\widetilde{V}$ is the disjoint union of a continuous family 
of $\widetilde{W}^{u}$-discs of the type of $\widetilde{W}_{l o c}^{u}(\tilde{x})$ as introduced by (1.3). For $\tilde{x} \in \widetilde{V}$, let $\widetilde{V}_{\tilde{x}}$ denote the $\widetilde{W}^{u}$-disc in $\widetilde{V}$ containing $\tilde{x}$, and $\tilde{\mu}_{\tilde{x}}$ the conditional probability measure of $\tilde{\mu}$ on $\widetilde{V}_{\tilde{x}}$. We say that $\mu$ has the SRB property if for every $\widetilde{V}$ as given above one has $\pi\left(\tilde{\mu}_{\tilde{x}}\right) \ll \lambda_{\tilde{x}}^{u}$ for $\tilde{\mu}$-a.e. $\tilde{x} \in \tilde{V}$, where $\pi\left(\tilde{\mu}_{\tilde{x}}\right)$ is the projection of $\tilde{\mu}_{\tilde{x}}$ under $\left.\pi\right|_{\widetilde{V}_{\tilde{x}}}: \widetilde{V}_{\tilde{x}} \rightarrow W_{l o c}^{u}(\tilde{x})$ and $\lambda_{\tilde{x}}^{u}$ is the Lebesgue measure on $W_{l o c}^{u}(\tilde{x})$ induced by its inherited Riemannian structure as a submanifold of $M$.

Now we give a precise definition of the SRB property.

Definition 1.2. We say that the $f$-invariant measure $\mu$ has the SRB property, if for every measurable partition $\eta$ of $M^{f}$ subordinate to $W^{u}$-manifolds of $(f, \mu)$ we have, for $\tilde{\mu}$-a.e. $\tilde{x} \in M^{f}$,

$$
\pi\left(\tilde{\mu}_{\tilde{x}}^{\eta}\right)<<\lambda_{\tilde{x}}^{u}
$$

where $\left\{\tilde{\mu}_{\tilde{x}}^{\eta}\right\}_{\tilde{x} \in M^{f}}$ is a canonical system of conditional measures of $\tilde{\mu}$ associated with $\eta, \pi\left(\tilde{\mu}_{\tilde{x}}^{\eta}\right)$ is the projection of $\tilde{\mu}_{\tilde{x}}^{\eta}$ under $\left.\pi\right|_{\eta(\tilde{x})}$ and $\lambda_{\tilde{x}}^{u}$ denotes the Lebesgue measure on $W_{\tilde{x}}$ induced by its inherited Riemannian structure as a submanifold of $M\left(\lambda_{\tilde{x}}^{u}=\delta_{x_{0}}\right.$ if $\left.W^{u}(\tilde{x})=\left\{x_{0}\right\}\right)$.

Remark 1.1. The definition of the SRB property of $\mu$ is clearly independent of the choice of the submanifolds $W_{\tilde{x}}, \tilde{x} \in M^{f}$.

The main result of this paper is the following theorem.

Theorem 1.1. Let $f$ be a $C^{2}$ endomorphism on $M$ with an $f$-invariant Borel probability measure $\mu$ satisfying the integrability condition (1.6). Then the entropy formula

$$
h_{\mu}(f)=\int_{M} \sum_{i} \lambda^{(i)}(x)^{+} m_{i}(x) d \mu(x)
$$

holds if and only if $\mu$ has the SRB property.

Let $f: M \rightarrow M$ be as given above and let $\mu$ be an $f$-invariant Borel probability measure. It is proved by Thieullen [17] and Liu 7] that, if $\mu$ is absolutely continuous with respect to the Lebesgue measure on $M$, then the integrability condition (1.6) and entropy formula (1.7) hold. Then, as a consequence of Theorem 1.1, we have the following corollary.

Corollary 1.1.1. Let $f$ be a $C^{2}$ endomorphism on $M$ with an invariant Borel probability measure $\mu$. If $\mu$ is absolutely continuous with respect to the Lebesgue measure on $M$, then $\mu$ has the SRB property.

Remark 1.2. In the diffeomorphism case, this corollary follows from the absolute continuity of the unstable foliation. But in the endomorphism case, it seems at least very difficult to prove the corollary along this line.

As a corollary of Theorem A of [13] and our Theorem 1.1, invariant measures of Axiom A attractors of $C^{2}$ endomorphisms also have the SRB property. We now state this result in a more detailed way. Let $O \subset M$ be an open set and let $f \in C^{2}(O, M)$. Assume that $\Lambda \subset O$ is closed and $f$-invariant (i.e., $f \Lambda=\Lambda$ ). Define

$$
\Lambda^{f}=\left\{\tilde{x}=\left\{x_{i}\right\}_{i \in \mathbb{Z}} \mid x_{i} \in \Lambda, f\left(x_{i}\right)=x_{i+1}, \forall i \in \mathbb{Z}\right\}
$$

and let $E=\pi^{*} T_{\Lambda} M$ be the pull-back bundle of the tangent bundle $T_{\Lambda} M$ via the natural projection $\pi: \Lambda^{f} \rightarrow \Lambda$. $\Lambda$ is called hyperbolic if there is a $T f$-invariant 
Whitney splitting $E=E^{s} \oplus E^{u}$ such that $\left.T f\right|_{E^{s}}$ is contracting and $\left.T f\right|_{E^{u}}$ is expanding (see [13] for a detailed definition). A hyperbolic set $\Lambda$ is called an Axiom $A$ attractor if (1) the periodic set of $f$ is dense in $\Lambda,(2)\left\{f^{i}\left(x_{0}\right)\right\}_{i=0}^{+\infty}$ is dense in $\Lambda$ for some $x_{0} \in \Lambda$, and (3) there exists an arbitrarily small open neighborhood $U$ of $\Lambda$ such that $f \bar{U} \subset U$ and $\bigcap_{n=0}^{+\infty} f^{n} U=\Lambda$. For $\tilde{x} \in \Lambda^{f}$, define

$$
\left\{\tilde{y}=\left\{y_{n}\right\}_{n \in \mathbb{Z}} \mid y_{n} \in O, f y_{n}=y_{n+1}, \varlimsup_{n \rightarrow+\infty} \frac{1}{n} \log d\left(x_{-n}, y_{-n}\right)<0\right\} .
$$

From the definition it follows easily that, if $\Lambda$ is an Axiom A attractor of $f$, then

$$
\widetilde{W}^{u}(\tilde{x}) \subset \Lambda^{f}
$$

and

$$
W^{u}(\tilde{x}):=\pi \widetilde{W}^{u}(\tilde{x}) \subset \Lambda
$$

for all $\tilde{x} \in \Lambda^{f}$. Hence an Axiom A attractor is the union of a family of unstable manifolds. The following result follows directly from Theorem A of [13] and our Theorem 1.1.

Corollary 1.1.2. Let $\Lambda$ be an Axiom A attractor of $f \in C^{2}(O, M)$ and assume that $T_{x} f$ is nondegenerate for every $x \in \Lambda$. Then there exists a unique $f$-invariant Borel probability measure $\mu$ on $\Lambda$ which is characterized by each of the following properties:

(1) $\mu$ has the SRB property.

(2) The system $f:(\Lambda, \mu) \rightarrow(M, \mu)$ satisfies the entropy formula (1.7).

(3) When $\varepsilon>0$ is small enough, $\frac{1}{n} \sum_{k=0}^{n-1} \delta_{f^{k} x}$ converges to $\mu$ as $n \rightarrow+\infty$ for Lebesque almost every $x \in B_{\varepsilon}(\Lambda):=\{y \in M \mid d(y, \Lambda)<\varepsilon\}$.

\section{Preliminaries}

In this section, we present some preliminary results which will be useful in the subsequent sections. Instead of giving the detailed proofs of these results, we will point out the relevent references. Readers familiar with 8 ] will see later on that, once these results are introduced, many arguments for the proof of Theorem 1.1 will be close to those in the case of random diffeomorphisms ([8, Chapter VI]) and hence the presentation can be simplified.

Throughout what follows, we will always assume that $f$ is a $C^{2}$ endomorphism on $M$ and $\mu$ is an $f$-invariant Borel probability measure satisfying Condition (1.6). Let $\tilde{\mu}$ be the $\tau$-invariant Borel probability measure on $M^{f}$ corresponding to $\mu$.

It is easy to see that there is a Borel set $\Lambda$ such that $f \Lambda \subset \Lambda$ and for every $x \in \Lambda$, $T_{x} f$ is nondegenerate and $\lambda^{s(x)}(x)>-\infty$, where $\lambda^{s(x)}$ is the smallest Lyapunov exponent of $f$ at $x$. It is also clear that condition (1.6) implies $\mu(\Lambda)=1$. Put

$$
\Lambda^{f}=\left\{\tilde{x} \in M^{f} \mid x_{i} \in \Lambda, \forall i \in \mathbb{Z}\right\}
$$

which obviously satisfies $\tau\left(\Lambda^{f}\right)=\Lambda^{f}$ and $\tilde{\mu}\left(\Lambda^{f}\right)=1$.

Let $E=\pi^{*} T M$ be the pull-back bundle of the tangent bundle $T M$ via the projection $\pi: M^{f} \rightarrow M$. If we define $A(\tilde{x})=T_{x_{0}} f$ for $\tilde{x} \in \Lambda^{f}$, then from (1.6) it follows that

$$
\int_{\Lambda^{f}}\left[\log ^{+}|A(\tilde{x})|+\log ^{+}\left|A(\tilde{x})^{-1}\right|\right] d \tilde{\mu}(\tilde{x})<+\infty .
$$


Applying the Oseledec multiplicative ergodic theorem presented in Appendix 2 of [3] to the obvious lifting to $E$ of $T f: T_{\Lambda} M \rightarrow T_{\Lambda} M$, one easily obtains the following fundamental result.

Proposition 2.1. There exists a Borel set $\tilde{\triangle} \subset \Lambda^{f}$ such that $\tau \tilde{\triangle}=\tilde{\triangle}$ and $\tilde{\mu}(\tilde{\triangle})=$ 1. Futrthermore, for every $\tilde{x} \in \tilde{\triangle}$ there exist a measurable (in $\tilde{x}$ ) splitting of the tangent space $T_{x_{0}} M$

$$
T_{x_{0}} M=E^{(1)}(\tilde{x}) \oplus E^{(2)}(\tilde{x}) \oplus \cdots \oplus E^{(s(\tilde{x}))}(\tilde{x})
$$

and measurable (in $\tilde{x}$ ) numbers

$$
+\infty>\lambda^{(1)}(\tilde{x})>\lambda^{(2)}(\tilde{x})>\cdots>\lambda^{(s(\tilde{x}))}(\tilde{x})>-\infty
$$

such that:

(1) Define, for $m \in \mathbb{Z}$,

$$
T^{m}(\tilde{x})= \begin{cases}T_{x_{0}} f^{m}, & \text { if } m>0, \\ \text { id, } & \text { if } m=0, \\ \left(T_{x_{m}} f^{-m}\right)^{-1}, & \text { if } m<0 .\end{cases}
$$

Then,

$$
\lim _{m \rightarrow \pm \infty} \frac{1}{m} \log \left|T^{m}(\tilde{x}) \xi\right|=\lambda^{(i)}(\tilde{x})
$$

for all $0 \neq \xi \in E^{(i)}(\tilde{x}), 1 \leq i \leq s(\tilde{x})$.

(2) $s(\tilde{x})$ and $\lambda^{(i)}(\tilde{x})$ are $\tau$-invariant, i.e., $s(\tau \tilde{x})=s(\tilde{x})$ and $\lambda^{(i)}(\tau \tilde{x})=\lambda^{(i)}(\tilde{x})$, $i=1, \ldots, s(\tilde{x}) . E^{(i)}(\tilde{x})$ is Tf-invariant, i.e., $T_{x_{0}} f E^{(i)}(\tilde{x})=E^{(i)}(\tau \tilde{x})$ for each $1 \leq i \leq s(\tilde{x})$. Moreover, $s(\tilde{x})=s\left(x_{0}\right), \lambda^{(i)}(\tilde{x})=\lambda^{(i)}\left(x_{0}\right)$ and $m^{(i)}(\tilde{x}):=$ $\operatorname{dim} E^{(i)}(\tilde{x})=m_{i}\left(x_{0}\right)$ for all $1 \leq i \leq s(\tilde{x})$, where $s\left(x_{0}\right), \lambda^{(i)}\left(x_{0}\right)$ and $m_{i}\left(x_{0}\right)$ are as introduced in the previous section.

(3) We introduce

$$
\rho^{(1)}(\tilde{x}) \geq \rho^{(2)}(\tilde{x}) \geq \cdots \geq \rho^{\left(m_{0}\right)}(\tilde{x})
$$

to denote $\lambda^{(1)}(\tilde{x}), \ldots, \lambda^{(1)}(\tilde{x}), \ldots, \lambda^{(i)}(\tilde{x}), \ldots, \lambda^{(i)}(\tilde{x}), \ldots, \lambda^{(s(\tilde{x}))}(\tilde{x})$, $\ldots, \lambda^{(s(\tilde{x}))}(\tilde{x})$, with $\lambda^{(i)}(\tilde{x})$ being repeated $m^{(i)}(\tilde{x})$ times. Now if $\left\{\xi_{1}, \ldots\right.$, $\left.\xi_{m_{0}}\right\}$ is a basis of $T_{x_{0}} M$ satisfying

$$
\lim _{m \rightarrow \pm \infty} \frac{1}{m} \log \left|T^{m}(\tilde{x}) \xi_{i}\right|=\rho^{(i)}(\tilde{x}) \quad \text { for every } \quad 1 \leq i \leq m_{0},
$$

then for any two non-empty disjoint subsets $P, Q \subset\left\{1, \cdots, m_{0}\right\}$ we have

$$
\lim _{m \rightarrow \pm \infty} \frac{1}{m} \log \gamma\left(T^{m}(\tilde{x}) E_{P}, T^{m}(\tilde{x}) E_{Q}\right)=0
$$

where $E_{P}$ and $E_{Q}$ denote respectively the subspaces of $T_{x_{0}} M$ spanned by the vectors $\left\{\xi_{i}\right\}_{i \in P}$ and $\left\{\xi_{j}\right\}_{j \in Q}$, and $\gamma(\cdot, \cdot)$ denotes the angle between the two associated subspaces.

The following proposition (a proof can be found in [19]) is very useful for dealing with unstable manifolds of $f$ and for constructing Lyapunov charts as done in $[8$, Section VI.3]. For $x \in M$ and $r>0$, we define $T_{x} M(r)=\left\{\xi \in T_{x} M|| \xi \mid<r\right\}$.

Proposition 2.2. There exists a Borel set $\triangle_{0} \subset \tilde{\triangle}$ with $\mu\left(\triangle_{0}\right)=1$ and $\tau \triangle_{0}=\triangle_{0}$ such that for any given $\varepsilon>0$ one can define a measurable function $\tilde{r}: \triangle_{0} \rightarrow$ $[1,+\infty)$ with the following properties. 
(1) For any $\tilde{x} \in \triangle_{0}$ there exists an open neighborhood of the zero vector $V_{x_{-1}}$ in $T_{x_{-1}} M$ such that the map

$$
H_{x_{-1}}:=\exp _{x_{0}}^{-1} \circ f \circ \exp _{x_{-1}}: V_{x_{-1}} \rightarrow T_{x_{0}} M\left(\tilde{r}(\tilde{x})^{-1}\right)
$$

is a diffeomorphism and the inverse map

$$
G_{\tilde{x}}:=H_{x_{-1}}^{-1}: T_{x_{0}} M\left(\tilde{r}(\tilde{x})^{-1}\right) \rightarrow V_{x_{-1}}
$$

satisfies

$$
\operatorname{Lip}\left(T \cdot G_{\tilde{x}}\right) \leq \tilde{r}(\tilde{x}),
$$

where T. $G_{\tilde{x}}: \xi \mapsto T_{\xi} G_{\tilde{x}}, \xi \in T_{x_{0}} M\left(\tilde{r}(\tilde{x})^{-1}\right)$.

(2) $\tilde{r}\left(\tau^{-n} \tilde{x}\right) \leq \tilde{r}(\tilde{x}) e^{\varepsilon n}, n \in \mathbb{Z}^{+}, \tilde{x} \in \triangle_{0}$.

We may assume that every point $\tilde{x} \in \triangle_{0}$ is regular in the sense of Proposition 2.1. For $\tilde{x} \in \triangle_{0}$, put

$$
E^{u}(\tilde{x})=\bigoplus_{\lambda^{(i)}(\tilde{x})>0} E^{(i)}(\tilde{x}), \quad E^{s c}(\tilde{x})=\bigoplus_{\lambda^{(i)}(\tilde{x}) \leq 0} E^{(i)}(\tilde{x})
$$

and

$$
\triangle_{0}^{\prime}=\left\{\tilde{x} \in \triangle_{0} \mid \lambda^{(i)}(\tilde{x})>0 \text { for some } 1 \leq i \leq s(\tilde{x})\right\} .
$$

The following proposition is a very useful result concerning local and global unstable manifolds of $f$ (see [19] for a proof).

Proposition 2.3. There are a countable number of compact subsets $\triangle_{i}, i \in \mathbb{N}$, of $M^{f}$ with $\bigcup_{i \in \mathbb{N}} \triangle_{i} \subset \triangle_{0}^{\prime}$ and $\tilde{\mu}\left(\triangle_{0}^{\prime} \backslash \bigcup_{i \in \mathbb{N}} \triangle_{i}\right)=0$ such that:

(1) For each $\triangle_{i}$, $\operatorname{dim} E^{u}(\tilde{x})=$ const., written $k_{i}$, for all $\tilde{x} \in \Lambda_{i} ; E^{u}(\tilde{x})$ and $E^{s c}(\tilde{x})$ depend continuously on $\tilde{x} \in \Lambda_{i}$.

(2) For any $\triangle_{i}$, there is a continuous family of $C^{1,1}$ embedded $k_{i}$-dimensional discs $\left\{W_{\text {loc }}^{u}(\tilde{x})\right\}_{\tilde{x} \in \triangle_{i}}$ in $M$ together with positive numbers $\lambda_{i}, \varepsilon_{i}<\lambda_{i} / 200$, $r_{i}<1, \gamma_{i}, \alpha_{i}$ and $\beta_{i}$ such that the following hold for each $\tilde{x} \in \triangle_{i}$ :

(i) There is a $C^{1,1}$ map

$$
h_{\tilde{x}}: O_{\tilde{x}} \rightarrow E^{s c}(\tilde{x}),
$$

where $O_{\tilde{x}}$ is an open subset of $E^{u}(\tilde{x})$ which contains $\left\{\xi \in E^{u}(\tilde{x})|| \xi \mid<\right.$ $\left.\alpha_{i}\right\}$, satisfying

(a) $h_{\tilde{x}}(0)=0, T_{0} h_{\tilde{x}}=0$;

(b) $\operatorname{Lip}\left(h_{\tilde{x}}\right) \leq \beta_{i}, \operatorname{Lip}\left(T \cdot h_{\tilde{x}}\right) \leq \beta_{i}$, where $T \cdot h_{\tilde{x}}: \xi \mapsto T_{\xi} h_{\tilde{x}}$;

(c) $W_{l o c}^{u}(\tilde{x})=\exp _{x_{0}} \operatorname{Graph}\left(h_{\tilde{x}}\right) \subset B\left(x_{0}, \tilde{r}(\tilde{x})^{-1}\right)$.

(ii) For any $y_{0} \in W_{\text {loc }}^{u}(\tilde{x})$ there exists a unique $\tilde{y} \in M^{f}$ such that $\pi \tilde{y}=y_{0}$,

$$
d\left(y_{-n}, x_{-n}\right) \leq r_{i} e^{-\varepsilon_{i} n}, \quad \forall n \in \mathbb{N},
$$

and

$$
d\left(y_{-n}, x_{-n}\right) \leq \gamma_{i} e^{-\lambda_{i} n}, \quad \forall n \in \mathbb{N} .
$$

(iii) There is a sequence $\left\{W_{-n}(\tilde{x})\right\}_{n=0}^{+\infty}$ of $C^{1,1}$ embedded $k_{i}$-dimensional discs in $M$ such that $W_{0}(\tilde{x})=W_{\text {loc }}^{u}(\tilde{x}), f W_{-n}(\tilde{x}) \supset W_{-n+1}(\tilde{x})$ for all $n \geq 1$ and

$$
W^{u}(\tilde{x})=\bigcup_{n=0}^{+\infty} f^{n} W_{-n}(\tilde{x})
$$


where $W^{u}(\tilde{x})$ is defined by 1.4. Moreover,

$$
\widetilde{W}^{u}(\tilde{x})=\left\{\tilde{y} \in M^{f} \mid \varlimsup_{n \rightarrow+\infty} \frac{1}{n} \log d\left(y_{-n}, x_{-n}\right) \leq-\lambda^{(p)}(\tilde{x})\right\},
$$

where $\lambda^{(p)}(\tilde{x})$ is the smallest strictly positive Lyapumov exponent of $f$ at $\tilde{x}$ and $\widetilde{W}^{u}(\tilde{x})$ is defined by (1.5).

(iv) Let $\widetilde{W}_{\text {loc }}^{u}(\tilde{x})$ denote the set of points $\tilde{y}$ as introduced in (ii) above. Then for any $\tilde{y}, \tilde{z} \in \widetilde{W}_{l o c}^{u}(\tilde{x})$, one has $y_{-n}, z_{-n} \in W_{-n}(\tilde{x})$ and

$$
d^{u}\left(y_{-n}, z_{-n}\right) \leq \gamma_{i} e^{-\lambda_{i} n}
$$

for all $n \in \mathbb{Z}^{+}$, where $d^{u}($,$) is the distance along W_{-n}(\tilde{x}), n \in \mathbb{Z}^{+}$.

(v) For any $\tilde{y} \in \widetilde{W}^{u}(\tilde{x})$,

$$
\varlimsup_{n \rightarrow+\infty} \frac{1}{n} \log d^{u}\left(y_{-n}, x_{-n}\right) \leq-\lambda^{(p)}(\tilde{x}),
$$

where $d^{u}\left(y_{-n}, x_{-n}\right)$ is the distance between $y_{-n}$ and $x_{-n}$ along $W_{-n}(\tilde{x})$ if $y_{-n} \in W_{-n}(\tilde{x})$, and $d^{u}\left(y_{-n}, x_{-n}\right)=1$ if $y_{-n} \notin W_{-n}(\tilde{x})$.

From the conclusion of Proposition 2.3 one can easily see that the local unstable manifolds $W_{l o c}^{u}(\tilde{x})$ have some additional properties, which we summarize in the next proposition. Let $\rho_{0}>0$ be a positive real number such that the exponential map $\exp _{x}: T_{x} M\left(\rho_{0}\right) \rightarrow B\left(x, \rho_{0}\right):=\left\{y \in M \mid d(y, x)<\rho_{0}\right\}$ is a $C^{\infty}$ diffeomorphism for every $x \in M$.

Proposition 2.4. Let $\triangle_{i},\left\{W_{l o c}^{u}(\tilde{x})\right\}_{\tilde{x} \in \triangle_{i}}$ and $\left\{\widetilde{W}_{l o c}^{u}(\tilde{x})\right\}_{\tilde{x} \in \triangle_{i}}, i \in \mathbb{N}$, be as introduced in Proposition 2.3. Then for any $\triangle_{i}$ there exist numbers $r_{i} \in\left(0, \rho_{0} / 4\right)$, $\varepsilon_{i} \in(0,1)$ and $R_{i}>0$ such that the following statements hold.

(1) For any $r \in\left[r_{i} / 2, r_{i}\right]$ and each $\tilde{x} \in \triangle_{i}$, if $\tilde{x}^{\prime} \in \widetilde{B}_{\triangle_{i}}\left(\tilde{x}, \varepsilon_{i} r\right):=\left\{\tilde{y} \in \triangle_{i} \mid\right.$ $\left.d(\tilde{x}, \tilde{y})<\varepsilon_{i} r\right\}$, then $W_{l o c}^{u}\left(\tilde{x}^{\prime}\right) \cap B\left(x_{0}, r\right)$ is connected and the map

$$
\tilde{x}^{\prime} \mapsto W_{l o c}^{u}\left(\tilde{x}^{\prime}\right) \cap B\left(x_{0}, r\right)
$$

is continuous from $\widetilde{B}_{\triangle_{i}}\left(\tilde{x}, \varepsilon_{i} r\right)$ to the space of subsets of $B\left(x_{0}, r\right)$ (endowed with the Hausdorff topology);

(2) Let $r \in\left[r_{i} / 2, r_{i}\right]$ and $\tilde{x} \in \Lambda_{i}$. If $\tilde{x}^{\prime}$ and $\tilde{x}^{\prime \prime} \in \widetilde{B}_{\Lambda_{i}}\left(\tilde{x}, \varepsilon_{i} r\right)$, then either

$$
W_{l o c}^{u}\left(\tilde{x}^{\prime}\right) \cap B\left(x_{0}, r\right)=W_{l o c}^{u}\left(\tilde{x}^{\prime \prime}\right) \cap B\left(x_{0}, r\right)
$$

or the two terms in the above equation are disjoint. In the latter case, if it is assumed moreover that $\tilde{x}^{\prime \prime} \in \widetilde{W}^{u}\left(\tilde{x}^{\prime}\right)$, then

$$
d^{u}\left(y_{0}, z_{0}\right)>2 r_{i}
$$

for any $y_{0} \in W_{\text {loc }}^{u}\left(\tilde{x}^{\prime}\right) \cap B\left(x_{0}, r\right)$ and any $z_{0} \in W_{\text {loc }}^{u}\left(\tilde{x}^{\prime \prime}\right) \cap B\left(x_{0}, r\right)$, where $d^{u}(\cdot, \cdot)$ is the distance along $W^{u}(\tilde{x})$, i.e., $d^{u}\left(y_{0}, z_{0}\right)$ is the distance between $y_{0}$ and $z_{0}$ along $W_{\mathrm{loc}}^{u}\left(\tilde{x}^{\prime}\right)$ if $y_{0}, z_{0} \in W_{\text {loc }}^{u}\left(\tilde{x}^{\prime}\right)$, and otherwise $d^{u}\left(y_{0}, z_{0}\right)=1$.

(3) For each $\tilde{x} \in \triangle_{i}$, if $\tilde{x}^{\prime} \in \widetilde{B}_{\triangle_{i}}\left(\tilde{x}, \varepsilon_{i} r\right)$ and $y_{0} \in W_{l o c}^{u}\left(\tilde{x}^{\prime}\right) \cap B\left(x_{0}, r\right)$, then $W_{l o c}^{u}\left(\tilde{x}^{\prime}\right)$ contains the closed ball of center $y_{0}$ and $d^{u}$ radius $R_{i}$ in $W^{u}\left(\tilde{x}^{\prime}\right)$. 


\section{Technical Preparations}

We now prepare to use the local unstable sets $\widetilde{W}_{l o c}^{u}(\tilde{x})$ of $f$ in $M^{f}$ to construct a suitable measurable partition of $M^{f}$ subordinate to $W^{u}$-manifolds of $f$. Notice that the partition of $M^{f}$ into global unstable sets $\left\{\widetilde{W}^{u}(\tilde{x})\right\}_{\tilde{x} \in M^{f}}$ is in general not measurable, but we may consider the $\sigma$-algebra consisting of measurable subsets of $M^{f}$ which are unions of some global unstable sets, i.e., the $\sigma$-algebra

$$
\mathcal{B}^{u}=\left\{B \in \mathcal{B}_{\tilde{\mu}}\left(M^{f}\right) \mid \tilde{x} \in B \text { implies } \widetilde{W}^{u}(\tilde{x}) \subset B\right\},
$$

where $\mathcal{B}_{\tilde{\mu}}\left(M^{f}\right)$ is the completion of $\mathcal{B}\left(M^{f}\right)$ with respect to $\tilde{\mu}$. In addition, put

$$
\mathcal{B}^{I}=\left\{A \in \mathcal{B}_{\tilde{\mu}}\left(M^{f}\right) \mid \tau^{-1} A=A\right\} .
$$

We have $\mathcal{B}^{I} \subset \mathcal{B}^{u}, \tilde{\mu}$-mod 0 .

In general, we have the following useful fact.

Lemma 3.1. Let $(X, d)$ be a compact metric space, and $T$ a homeomorphism with an invariant probability measure $\mu$. Let

$$
\mathcal{B}^{I}(X, T, \mu)=\left\{A \in \mathcal{B}_{\mu}(X) \mid T^{-1} A=A\right\}
$$

and

$$
\mathcal{B}^{u}(X, T, \mu)=\left\{B \in \mathcal{B}_{\mu}(X) \mid x \in B \text { implies } W^{u}(x) \subset B\right\}
$$

where

$$
W^{u}(x)=\left\{y \in X \mid \lim _{n \rightarrow+\infty} d\left(T^{-n} y, T^{-n} x\right)=0\right\} .
$$

Then $\mathcal{B}^{I}(X, T, \mu) \subset \mathcal{B}^{u}(X, T, \mu), \mu$-mod 0 .

Proof. For simplicity, write $\mathcal{B}^{u}(X)=\mathcal{B}^{u}(X, T, \mu)$ and $\mathcal{B}^{I}(X)=\mathcal{B}^{I}(X, T, \mu)$.

Since $X$ is a compact metric space, there exists a countable set $\mathcal{F} \subset C(X)$ which is dense in $L^{2}\left(X, \mathcal{B}_{\mu}(X), \mu\right)$. For any $g \in \mathcal{F}$, according to the Birkhoff ergodic theorem and the general properties of conditional expectations, one has

$$
\lim _{n \rightarrow+\infty} \frac{1}{n} \sum_{k=0}^{n-1} g\left(T^{-k} x\right)=E^{\mu}\left[g \mid \mathcal{B}^{I}(X)\right](x)
$$

for every point $x$ of a set $\Lambda_{g} \in \mathcal{B}^{I}(X)$ with $\mu\left(\Lambda_{g}\right)=1$. Denote $\Lambda_{\mathcal{F}}=\bigcap_{g \in \mathcal{F}} \Lambda_{g}$. Since $\mathcal{F}$ is countable, we have $\mu\left(\Lambda_{\mathcal{F}}\right)=1$. If two points $y, z \in \Lambda_{\mathcal{F}}$ belong to the same unstable set, i.e. there exists $x$ such that $y, z \in W^{u}(x)$, then by (3.3) we have, for each $g \in \mathcal{F}$,

$$
E^{\mu}\left[g \mid \mathcal{B}^{I}(X)\right](y)=E^{\mu}\left[g \mid \mathcal{B}^{I}(X)\right](z),
$$

since $d\left(T^{-n} y, T^{-n} z\right) \rightarrow 0$ as $n \rightarrow+\infty$. Therefore, each $\left.E^{\mu}\left[g \mid \mathcal{B}^{I}(X)\right]\right|_{\Lambda_{\mathcal{F}}}$ (the restriction of $E^{\mu}\left[g \mid \mathcal{B}^{I}(X)\right]$ to $\left.\Lambda_{\mathcal{F}}\right)$ is measurable with respect to $\left.\mathcal{B}^{u}(X)\right|_{\Lambda_{\mathcal{F}}}$, and hence

$$
\left\{\left.E^{\mu}\left[g \mid \mathcal{B}^{I}(X)\right]\right|_{\Lambda_{\mathcal{F}}} \mid g \in \mathcal{F}\right\} \subset L^{2}\left(\Lambda_{\mathcal{F}},\left.\mathcal{B}^{u}(X)\right|_{\Lambda_{\mathcal{F}}}, \mu\right) .
$$

Since $\mathcal{F}$ is a dense subset of $L^{2}\left(X, \mathcal{B}_{\mu}(X), \mu\right),\left\{E^{\mu}\left[g \mid \mathcal{B}^{I}(X)\right] \mid g \in \mathcal{F}\right\}$ is dense in $L^{2}\left(X, \mathcal{B}^{I}(X), \mu\right)$. Then from (3.4) it follows that

$$
L^{2}\left(\Lambda_{\mathcal{F}},\left.\mathcal{B}^{I}(X)\right|_{\Lambda_{\mathcal{F}}}, \mu\right) \subset L^{2}\left(\Lambda_{\mathcal{F}},\left.\mathcal{B}^{u}(X)\right|_{\Lambda_{\mathcal{F}}}, \mu\right),
$$


which implies

$$
\mathcal{B}^{I}(X, T, \mu) \subset \mathcal{B}^{u}(X, T, \mu), \quad \mu-\bmod 0,
$$

$\operatorname{as} \mu\left(\Lambda_{\mathcal{F}}\right)=1$.

As a consequence of Lemma 3.1, we have the following fact, which plays a crucial role in this section and even in the whole proof of Theorem 1.1

Corollary 3.1.1. $\mathcal{B}^{I} \subset \mathcal{B}^{u}, \tilde{\mu}-\bmod 0$.

A measurable partition $\eta$ of $M^{f}$ is said to be increasing if $\tau^{-1} \eta \geq \eta$. In what follows, we prove that there exists an increasing partition with a refinement subordinate to $W^{u}$-manifolds of $f$ and other good properties. Such partitions are very useful in our proof of the main theorem.

The main result of this section is the following proposition.

Proposition 3.2. There exists a measurable partition $\eta$ of $M^{f}$ which has the following properties:

(1) $\tau^{-1} \eta \geq \eta$.

(2) $\bigvee_{n=0}^{+\infty} \tau^{-n} \eta$ is equal to the partition into single points.

(3) $\mathcal{B}\left(\bigwedge_{n=0}^{+\infty} \tau^{n} \eta\right)=\mathcal{B}^{u}, \tilde{\mu}$-mod 0 , where $\mathcal{B}(\xi)$ is the $\sigma$-algebra consisting of all measurable $\xi$-sets for a measurable partition $\xi$ of $M^{f}$.

(4) There exists a measurable partition $\eta^{\prime} \geq \eta$ such that $\eta^{\prime}$ is subordinate to $W^{u}$-manifolds of $f$.

Proof. We prove the proposition by constructing such a partition.

Let $\Delta_{i} \in\left\{\Delta_{i} \mid i \in \mathbb{N}\right\}$ be fixed arbitrarily. Since $\Delta_{i}$ is compact, the open cover $\left\{\widetilde{B}_{\Delta_{i}}\left(\tilde{x}, \varepsilon_{i} r_{i} / 2\right)\right\}_{\tilde{x} \in \Delta_{i}}$ of $\Delta_{i}$ has a finite subcover, denoted by $\mathcal{U}_{\Delta_{i}}$. Pick arbitrarily a set $\widetilde{B}_{\Delta_{i}}\left(\tilde{x}^{*}, \varepsilon_{i} r_{i} / 2\right)$ from $\mathcal{U}_{\Delta_{i}}$. For each $r \in\left[r_{i} / 2, r_{i}\right]$ put

$$
S_{r}=\bigcup_{\tilde{x} \in \widetilde{B}_{\Delta_{i}}\left(\tilde{x}^{*}, \varepsilon_{i} r\right)}\left\{\tilde{y} \in \widetilde{W}_{l o c}^{u}(\tilde{x}) \mid y_{0} \in B\left(x_{0}^{*}, r\right)\right\},
$$

and assume that $\tilde{\mu}\left(S_{r}\right)>0$.

Let $\xi_{r}$ denote the partition of $M^{f}$ into all the sets $\left\{\tilde{y} \in M^{f} \mid y_{0} \in W_{l o c}^{u}(\tilde{x}) \cap\right.$ $\left.B\left(x_{0}^{*}, r\right)\right\}, \tilde{x} \in \widetilde{B}_{\Delta_{i}}\left(\tilde{x}^{*}, \varepsilon_{i} r\right)$ and the set $M^{f} \backslash S_{r}$. From Proposition 2.4, it is easy to see that $\xi_{r}$ is a measurable partition of $M^{f}$. Define

$$
\eta_{r}=\bigvee_{n=0}^{+\infty} \tau^{n} \xi_{r}, \quad \widehat{S}_{r}=\bigcup_{n=0}^{+\infty} \tau^{n} S_{r} .
$$

We claim that there exists $r \in\left[r_{i} / 2, r_{i}\right]$ such that $\eta_{r}$ has the following properties: (a) $\tau^{-1} \eta_{r} \geq \eta_{r}$.

(b) For $\tilde{\mu}$-a.e. $\tilde{y} \in \widehat{S}_{r}, \eta_{r}(\tilde{y}) \subset \widetilde{W}^{u}(\tilde{x})$ and $\pi\left(\eta_{r}(\tilde{y})\right)$ contains an open neighborhood of $y_{0}$ in $W_{l o c}^{u}(\tilde{y})$.

In fact, $\eta_{r}$ satisfies (a)-(b) for Lebesgue almost every $r \in\left[r_{i} / 2, r_{i}\right]$. We now prove this fact in several steps.

Step 1. From the definition of $\eta_{r}$ it follows clearly that $\eta_{r}$ satisfies (a) for each $r \in\left[r_{i} / 2, r_{i}\right]$. 
Step 2. Let $r \in\left[r_{i} / 2, r_{i}\right]$. It is clear that for any $\tilde{y} \in \widehat{S}_{r}$ one has $\eta_{r}(\tilde{y}) \subset \widetilde{W}^{u}(\tilde{y})$, since

$$
\eta_{r}(\tilde{y}) \subset \tau^{n} \widetilde{W}_{l o c}^{u}\left(\tau^{-n} \tilde{x}\right)
$$

for some $n \geq 0$ and some $\tau^{-n} \tilde{x} \in \widetilde{B}_{\Delta_{i}}\left(\tilde{x}^{*}, \varepsilon_{i} r\right)$.

On the other hand, we first claim that there exists a function $\beta_{r}: S_{r} \rightarrow \mathbb{R}^{+}$such that for any $\tilde{y} \in S_{r}, z_{0} \in W^{u}(\tilde{y})$ and $d^{u}\left(y_{0}, z_{0}\right) \leq \beta_{r}(\tilde{y})$ there is $\tilde{z} \in \eta_{r}(\tilde{y})$ with $\pi \tilde{z}=z_{0}$. Indeed, for $\tilde{y} \in S_{r}$ define

$$
\beta_{r}(\tilde{y})=\inf _{n \geq 0}\left\{R_{i}, \frac{1}{2 \gamma_{i}} d\left(y_{-n}, \partial B\left(x_{0}^{*}, r\right)\right) e^{n \lambda_{i}}, \frac{r}{\gamma_{i}}\right\} .
$$

From the definition of $S_{r}$, there is $\tilde{x} \in \widetilde{B}_{\Delta_{i}}\left(\tilde{x}^{*}, \varepsilon_{i} r\right)$ such that $\tilde{y} \in \widetilde{W}_{l o c}^{u}(\tilde{x})$. Since $d^{u}\left(y_{0}, z_{0}\right) \leq R_{i}$, by (3) of Proposition 2.4 there is $\tilde{z} \in \widetilde{W}_{\text {loc }}^{u}(\tilde{x})$ with $\pi \tilde{z}=z_{0}$, and hence for all $n \geq 0$

$$
d^{u}\left(y_{-n}, z_{-n}\right) \leq \gamma_{i} e^{-n \lambda_{i}} d^{u}\left(y_{0}, z_{0}\right) \leq \frac{1}{2} d\left(y_{-n}, \partial B\left(x_{0}^{*}, r\right)\right)
$$

and

$$
d^{u}\left(y_{-n}, z_{-n}\right) \leq \gamma_{i} e^{-n \lambda_{i}} \cdot \frac{r}{\gamma_{i}} \leq r .
$$

These together with (2) of Proposition 2.4 and the definition of $\xi_{r}$ easily imply that for all $n \geq 0$

$$
\tau^{-n} \tilde{z} \in \xi_{r}\left(\tau^{-n} \tilde{y}\right)
$$

which proves what we claimed just above.

We next claim that $\beta_{r}>0 \tilde{\mu}$-a.e. on $S_{r}$ for Lebesgue almost every $r \in\left[r_{i} / 2, r_{i}\right]$. In fact, let $\nu$ be the finite non-negative Borel measure on $\left[r_{i} / 2, r_{i}\right]$ defined by

$$
\nu(A)=\mu\left(\left\{x \in M \mid d\left(x, x_{0}^{*}\right) \in A\right\}\right)
$$

for each Borel subset $A$ of $\left[r_{i} / 2, r_{i}\right]$. According to [8, Lemma IV.2.1], Lebesgue almost every $r \in\left[r_{i} / 2, r_{i}\right]$ satisfies

$$
\sum_{n=0}^{+\infty} \mu\left(\left\{x \in M|| d\left(x, x_{0}^{*}\right)-r \mid<e^{-n \lambda_{i}}\right\}\right)<+\infty .
$$

Let $K_{0}=\left\{r \in\left[r_{i} / 2, r_{i}\right] \mid r\right.$ satisfies (3.7) and $\left.\mu\left(\partial B\left(x_{0}^{*}, r\right)\right)=0\right\}$. Clearly $\left|K_{0}\right|=$ $r_{i} / 2$. Let $r \in K_{0}$. By standard facts on Riemannian metrics, we have a constant $D>0$ such that the inequality

$$
d\left(x, \partial B\left(x_{0}^{*}, \rho\right)\right)<\tau
$$

implies

$$
\left|d\left(x, x_{0}^{*}\right)-\rho\right|<D \tau
$$

for $\rho$ and $\tau$ satisfying $0<\tau<\rho \leq r_{i}$. Thus from (3.7) we obtain

$$
\sum_{n=0}^{+\infty} \mu\left(\left\{x \in M \mid d\left(x, \partial B\left(x_{0}^{*}, r\right)\right)<D^{-1} e^{-n \lambda_{i}}\right\}\right)<+\infty .
$$


In other words,

$$
\sum_{n=0}^{+\infty} \tilde{\mu}\left(\left\{\tilde{x} \in M^{f} \mid d\left(x_{0}, \partial B\left(x_{0}^{*}, r\right)\right)<D^{-1} e^{-n \lambda_{i}}\right\}\right)<+\infty .
$$

This together with the $\tau$-invariance of $\tilde{\mu}$ yields that

$$
\sum_{n=0}^{+\infty} \tilde{\mu}\left(\left\{\tilde{y} \in M^{f} \mid d\left(y_{-n}, \partial B\left(x_{0}^{*}, r\right)\right)<D^{-1} e^{-n \lambda_{i}}\right\}\right)<+\infty .
$$

Then, by the Borel-Cantelli lemma, we know that $\tilde{\mu}$-a.e. $\tilde{y} \in M^{f}$ satisfies

$$
d\left(y_{-n}, \partial B\left(x_{0}^{*}, r\right)\right) \geq D^{-1} e^{-n \lambda_{i}}
$$

when $n$ is sufficiently large. Therefore, $\beta_{r}(\tilde{y})>0$ for $\tilde{\mu}$-a.e. $\tilde{y} \in S_{r}$. The second claim is then proved.

Let $r \in K_{0}$. The two claims above together imply that for $\tilde{\mu}$-a.e. $\tilde{y} \in S_{r}$, $\pi\left(\eta_{r}(\tilde{y})\right)$ contains an open neighborhood of $y_{0}$ in $W_{l o c}^{u}(\tilde{y})$. Furthermore, for every $n \geq 0$ we have

$$
\left.\eta_{r}\right|_{\tau^{n} S_{r}}=\left.\left[\left(\bigvee_{k=0}^{n-1} \tau^{k} \xi_{r}\right) \vee \tau^{n} \eta_{r}\right]\right|_{\tau^{n} S_{r}}=\left.\left(\bigvee_{k=0}^{n-1} \tau^{k} \xi_{r}\right)\right|_{\tau^{n} S_{r}} \vee \tau^{n}\left(\left.\eta_{r}\right|_{S_{r}}\right) .
$$

Since $\mu\left(\partial B\left(x_{0}^{*}, r\right)\right)=0$ implies $\tilde{\mu}\left(\left\{\tilde{y} \in M^{f} \mid y_{-l} \in \partial B\left(x_{0}^{*}, r\right)\right.\right.$ for some $\left.\left.l \geq 0\right\}\right)$ $=0$, from (3.8) it is easy to see that for $\tilde{\mu}$-a.e. $\tilde{y} \in \tau^{n} S_{r}, \eta_{r}(\tilde{y})$ contains an open neighborhood of $y_{0}$ in $W_{l o c}^{u}(\tilde{y})$. Thus $\eta_{r}$ satisfies the requirements in (b).

Put $I_{r}=\bigcap_{k=0}^{+\infty} \tau^{k} \widehat{S}_{r}$. Obviously $\tau^{-1} I_{r}=I_{r}$. By Corollary 3.1.1 we may assume that $I_{r} \in \mathcal{B}^{u}$, since otherwise there is $I_{r}^{\prime} \in \mathcal{B}^{u}$ with $\tau^{-1} I_{r}^{\prime}=I_{r}^{\prime}$ and $\tilde{\mu}\left(I_{r} \triangle I_{r}^{\prime}\right)=0$, and we may verify that $\eta_{r}$ restricted to $I_{r}$, written $\eta_{r}^{I}$, satisfies the requirements (2) and (3).

Put $\eta_{r}^{-}=\bigvee_{n=0}^{+\infty} \tau^{-n} \eta_{r}^{I}$. Since $\tau:\left(M^{f}, \tilde{\mu}\right) \rightarrow\left(M^{f}, \tilde{\mu}\right)$ restricted to $I_{r}$ is also measure-preserving, by Poincaré's recurrence theorem, for $\tilde{\mu}$-a.e. $\tilde{y} \in \widehat{S}_{r}$ there exist infinitely many positive integers $\left\{n_{j} \mid j=1,2, \cdots\right\}$ such that $\tau^{n_{j}} \tilde{y} \in S_{r}$ for all $j \geq 1$. Then, letting $d_{i}$ be the maximum $d^{u}$-diamter of the embedded discs introduced in Proposition 2.3 (2), we have that the $d^{u}$-diameter of $\pi\left(\eta_{r}^{-}(\tilde{y})\right)$ is less than $\gamma_{i} d_{i} e^{-\lambda_{i} n_{j}}$ for all $j \geq 1$ and hence is equal to 0 . This proves that $\eta_{r}^{-}$is equal to the partition of $I_{r}$ into single points.

In order to prove that $\left.\mathcal{B}\left(\bigwedge_{n=0}^{+\infty} \tau^{n} \eta_{r}^{I}\right) \subset \mathcal{B}^{u}\right|_{I_{r}}, \tilde{\mu}$-mod 0 , it suffices to ensure that for $\tilde{\mu}$-a.e. $\tilde{y} \in I_{r}$, if $\tilde{z} \in \widetilde{W}^{u}(\tilde{y})$, then there exists $k>0$ such that $\tau^{-k} \tilde{z} \in \eta_{r}^{I}\left(\tau^{-k} \tilde{y}\right)$. In fact, for $\tilde{\mu}$-a.e. $\tilde{y} \in I_{r}$, we first have

$$
\varlimsup_{n \rightarrow+\infty} \frac{1}{n} \log d^{u}\left(y_{-n}, z_{-n}\right) \leq-\lambda_{i}<0
$$

if $\tilde{z} \in \widetilde{W}^{u}(\tilde{y})$. Now define $\alpha_{r}: I_{r} \rightarrow \mathbb{R}^{+}$by

$$
\alpha_{r}(\tilde{y})= \begin{cases}\beta_{r}(\tilde{y}), & \text { if } \tilde{y} \in S_{r} ; \\ 0, & \text { otherwise. }\end{cases}
$$

According to Birkhoff's ergodic theorem, for $\tilde{\mu}$-a.e. $\tilde{y} \in I_{r}$ the limit

$$
\lim _{n \rightarrow+\infty} \frac{1}{n} \sum_{k=0}^{n-1} \alpha_{r}\left(\tau^{-k} \tilde{y}\right)=\alpha_{r}^{-}(\tilde{y})
$$


exists, and it is easy to see that $\alpha_{r}^{-}>0$ for $\tilde{\mu}$-a.e. $\tilde{y} \in I_{r}$. Hence, for $\tilde{\mu}$-a.e. $\tilde{y} \in I_{r}$, if $\tilde{z} \in \widetilde{W}^{u}(\tilde{y})$, there will be some $k>0$ such that

$$
d^{u}\left(y_{-k}, z_{-k}\right)<\alpha_{r}\left(\tau^{-k} \tilde{y}\right),
$$

which implies that $\tau^{-k} \tilde{y} \in S_{r}$ and $\tau^{-k} \tilde{z} \in \eta_{r}^{I}\left(\tau^{-k} \tilde{y}\right)$. On the other hand, it is clear that $\left.\mathcal{B}^{u}\right|_{I_{r}} \subset \mathcal{B}\left(\tau^{n} \eta_{r}^{I}\right)(\tilde{\mu}$-mod 0$)$ for all $n \geq 0$, and hence $\left.\mathcal{B}^{u}\right|_{I_{r}} \subset \bigcap_{n=0}^{+\infty} \mathcal{B}\left(\tau^{n} \eta_{r}^{I}\right)=$ $\mathcal{B}\left(\bigwedge_{n=0}^{+\infty} \tau^{n} \eta_{r}^{I}\right)(\tilde{\mu}$-mod 0$)$. This proves that $\eta_{r}^{I}$ satisfies the requirement (3).

Now notice that the treatment above holds for every element in the union $\bigcup_{i=1}^{+\infty} \mathcal{U}_{\Delta_{i}}=\left\{U_{1}, U_{2}, \cdots\right\}$. For each $U_{n}$ we denote by $\eta_{n}$ the associated partition $\eta_{r}$ constructed above satisfying (1)-(3), and by $I_{n}$ the corresponding $I_{r}$. Set $\hat{\eta}_{n}=\left.\eta_{n}\right|_{I_{n}}$ for $n \geq 1$ and define a partition $\eta$ of $M^{f}$ by

$$
\eta(\tilde{x})=\left\{\begin{array}{lll}
\hat{\eta}_{1}(\tilde{x}), & \text { if } & \tilde{x} \in I_{1} \\
\hat{\eta}_{n}(\tilde{x}), & \text { if } & \tilde{x} \in I_{n} \backslash \bigcup_{k=1}^{n-1} I_{k} ; \\
\{\tilde{x}\}, & \text { if } & \tilde{x} \in M^{f} \backslash \bigcup_{n=1}^{+\infty} I_{n} .
\end{array}\right.
$$

Since $I_{n} \in \mathcal{B}^{I} \cap \mathcal{B}^{u}$ for all $n \geq 1$ and $\tilde{\mu}\left(\Delta_{0}^{\prime} \backslash \bigcup_{n=1}^{+\infty} I_{n}\right)=0$, it is easy to see that $\eta$ satisfies the requirements (1)-(3).

To finish the proof of the proposition, for each $U_{n}$, we denote by $S_{n}$ the associated $S_{r}$ as defined in (3.5) for $r$ in the corresponding $K_{0}$. Let $\xi_{n}$ denote the corresponding partition $\xi_{r}$ of $M^{f}$, and define a partition $\xi$ of $M^{f}$ by

$$
\xi(\tilde{x})=\left\{\begin{array}{lll}
\xi_{1}(\tilde{x}), & \text { if } & \tilde{x} \in S_{1} ; \\
\xi_{n}(\tilde{x}), & \text { if } & \tilde{x} \in S_{n} \backslash \bigcup_{k=1}^{n-1} S_{k} ; \\
\{\tilde{x}\}, & \text { if } & \tilde{x} \in M^{f} \backslash \bigcup_{n=1}^{+\infty} S_{n} .
\end{array}\right.
$$

Since $\tilde{\mu}\left(\Delta_{0}^{\prime} \backslash \bigcup_{n=1}^{+\infty} S_{n}\right)=0$, it is easy to see that $\xi$ is subordinate to $W^{u}$-manifolds of $f$. Now take $\eta^{\prime}=\eta \vee \xi$; then $\eta^{\prime}$ satisfies the requirement (4).

The proof is completed.

Let $\eta$ and $\eta^{\prime}$ be as in the proof of Proposition 3.2 Clearly, for $\tilde{\mu}$-a.e. $\tilde{x} \in M^{f}$, $\left.\eta^{\prime}\right|_{\eta(\tilde{x})}$ is $\left(\tilde{\mu}_{\tilde{x}}^{\eta}\right.$-mod 0) a countable partition. Let $\eta(\tilde{x})=\bigcup_{i} A_{i},\left\{A_{1}, A_{2}, \cdots\right\} \subset \eta^{\prime}$. We know that for each $A_{i}$, there is a $C^{1}$ embedded submanifold $W_{i}$ of $M$ such that $\pi\left(A_{i}\right) \subset W_{i}$ and for $\tilde{\mu}$-a.e. $\tilde{y} \in M^{f}$, if $\tilde{y} \in A_{i}$, then

$$
\left.\pi\right|_{A_{i}}: A_{i} \rightarrow \pi\left(A_{i}\right)
$$

is bijective. Therefore, there is a unique measure $\tilde{\lambda}_{i}$ on $A_{i}$ such that

$$
\tilde{\lambda}_{i}(B)=\lambda_{i}(\pi(B))
$$

for every Borel set $B \subset A_{i}$, where $\lambda_{i}$ is the Lebesgue measure on $W_{i}$. Define $\tilde{\lambda}_{\tilde{x}}^{\eta}$ as

$$
\tilde{\lambda}_{\tilde{x}}^{\eta}(K)=\sum_{i} \tilde{\lambda}_{i}\left(K \cap A_{i}\right) \text {. }
$$

Proposition 3.3. For every Borel subset $B \subset M^{f}$, the function

$$
P_{B}(\tilde{x})=\tilde{\lambda}_{\tilde{x}}^{\eta}(\eta(\tilde{x}) \cap B)
$$

is measurable and $\tilde{\mu}$-almost everywhere finite.

Proof. Let $r \in K_{0}$ and $S_{r}$ be defined as in Step 1 of the proof of Proposition 3.2 From (3.6) it is easy to see that for any Borel subset $B \subset M^{f}$ the function $P_{B}(\tilde{y})$ is 
finite for $\tilde{\mu}$-a.e. $\tilde{y} \in \widehat{S}_{r}$. Let $n \in \mathbb{Z}^{+}$, and put $\xi_{r}^{n}=\bigvee_{k=0}^{n-1} \tau^{k} \xi_{r}$ and $\widehat{S}_{r}^{n}=\bigcup_{k=0}^{n-1} \tau^{k} S_{r}$. From the definition of $S_{r}$ it is clear that, if $U$ is an open ball in $M^{f}$, the function

$$
P_{U, n}(\tilde{y})=\tilde{\lambda}_{\tilde{y}}^{\eta}\left(\xi_{r}^{n}(\tilde{y}) \cap U\right)
$$

is measurable on $\widehat{S}_{r}^{n}$. Then the standard arguments from measure theory ensure that so is $P_{B, n}(\tilde{y})$ for any $B \in M^{f}$. Notice that for any Borel subset $B \in M^{f}$

$$
P_{B, n}(\tilde{y}) \geq P_{B, n+1}(\tilde{y})
$$

for each $\tilde{y} \in \widehat{S}_{r}^{n}$, and

$$
\lim _{n \rightarrow+\infty} P_{B, n}(\tilde{y})=P_{B}(\tilde{y})
$$

for $\tilde{\mu}$-a.e. $\tilde{y} \in \widehat{S}_{r}$. Therefore $P_{B}(\tilde{y})$ is measurable and finite $\tilde{\mu}$-a.e. on $\widehat{S}_{r}$. Then by the definition of $\eta$, we conclude that $P_{B}(\tilde{y})$ is measurable and finite $\tilde{\mu}$-a.e. on $M^{f}$.

Let $\eta$ be a partition as in Proposition 3.2. Proposition 3.2 allows us to define a Borel measure $\tilde{\lambda}^{*}$ on $M^{f}$ by

$$
\tilde{\lambda}^{*}(K)=\int \tilde{\lambda}_{\tilde{x}}^{\eta}(\eta(\tilde{x}) \cap K) d \tilde{\mu}(\tilde{x})
$$

for each Borel subset $K \subset M^{f}$. It is easy to see that $\tilde{\lambda}^{*}$ is $\sigma$-finite. Furthermore, by the definition of conditional measures we have

$$
\tilde{\mu}(K)=\int \tilde{\mu}_{\tilde{x}}^{\eta}(\eta(\tilde{x}) \cap K) d \tilde{\mu}(\tilde{x})
$$

for each Borel subset $K$ of $M^{f}$, where $\tilde{\mu}_{\tilde{x}}^{\eta}$ is regarded as the conditional measure of $\tilde{\mu}$ on $\eta(\tilde{x})$. If $\mu$ has the SRB property, one easily has

$$
\tilde{\mu} \ll \tilde{\lambda}^{*} \text {. }
$$

Define

$$
g=\frac{d \tilde{\mu}}{d \tilde{\lambda}^{*}} .
$$

By a measure-theoretic observation one has the following proposition.

Proposition 3.4. Suppose $\mu$ has the SRB property. Let $\eta$ be a partition as introduced in Proposition 3.2. Then for $\tilde{\mu}$-a.e. $\tilde{x}$ one has

$$
g=\frac{d \tilde{\mu}_{\tilde{x}}^{\eta}}{d \tilde{\lambda}_{\tilde{x}}^{\eta}}
$$

$\tilde{\lambda}_{\tilde{x}}^{\eta}$-almost everywhere on $\eta(\tilde{x})$, where $\tilde{\lambda}_{\tilde{x}}^{\eta}$ is given by (3.9).

Proof. Let us first notice that (3.10) can be written equivalently as

$$
\int \chi_{K} d \tilde{\lambda}^{*}=\int\left[\int_{\eta(\tilde{x})} \chi_{K}(\tilde{z}) d \tilde{\lambda}_{\tilde{x}}^{\eta}(\tilde{z})\right] d \tilde{\mu}(\tilde{x})
$$

for any Borel set $K \subset M^{f}$. Then, using standard methods of measure theory, we easily obtain

$$
\int h d \tilde{\lambda}^{*}=\int\left[\int_{\eta(\tilde{x})} h(\tilde{z}) d \tilde{\lambda}_{\tilde{x}}^{\eta}(\tilde{z})\right] d \tilde{\mu}(\tilde{x})
$$

for any $h \in L^{1}\left(M^{f}, \mathcal{B}\left(M^{f}\right), \tilde{\lambda}^{*}\right)$. 
Let $A \in \mathcal{B}(\eta), B \in \mathcal{B}\left(M^{f}\right)$ be two arbitrary sets. From (3.10)-(3.13) it follows that

$$
\begin{aligned}
\int_{A \cap B} g d \tilde{\lambda}^{*} & =\int_{A}\left[\int_{\eta(\tilde{x}) \cap B} g(\tilde{z}) d \tilde{\lambda}_{\tilde{x}}^{\eta}(\tilde{z})\right] d \tilde{\mu}(\tilde{x}) \\
& =\int_{A \cap B} d \tilde{\mu}=\int_{A} \tilde{\mu}_{\tilde{x}}^{\eta}(\eta(\tilde{x}) \cap B) d \tilde{\mu}(\tilde{x}) .
\end{aligned}
$$

Since $M^{f}$ is a Borel subset of the Polish space $M^{\mathbb{Z}}$, by [8, Theorem 0.1.3], the measure space $\left(M^{f}, \mathcal{B}\left(M^{f}\right), \tilde{\mu}\right)$ is separable. Then by [8, Theorem 0.1.2], $\mathcal{B}\left(M^{f}\right)$ can be generated $(\tilde{\mu}$-mod 0$)$ by a countable subalgebra $\left\{B_{i}\right\}_{i \in \mathbb{N}}$ of $\mathcal{B}\left(M^{f}\right)$. Fixing $i \in \mathbb{N}$, we apply (3.14) to an arbitrary set $A \in \mathcal{B}(\eta)$ and to $B=B_{i}$. As $A$ is arbitrary, (3.14) implies that there exists a measurable subset $Z_{i} \subset M^{f}$ such that $\tilde{\mu}\left(Z_{i}\right)=1$ and for each $\tilde{x} \in Z_{i}$ one has

$$
\int_{\eta(\tilde{x}) \cap B_{i}} g(\tilde{z}) d \tilde{\lambda}_{\tilde{x}}^{\eta}(\tilde{z})=\tilde{\mu}_{\tilde{x}}^{\eta}\left(\eta(\tilde{x}) \cap B_{i}\right) .
$$

Then, according to [ $\underline{8}$, Theorem 0.1.1], we know that for $\tilde{\mu}$-a.e. $\tilde{x}$ the equality

$$
\int_{\eta(\tilde{x}) \cap B} g(\tilde{z}) d \tilde{\lambda}_{\tilde{x}}^{\eta}(\tilde{z})=\tilde{\mu}_{\tilde{x}}^{\eta}(\eta(\tilde{x}) \cap B)
$$

holds for any $B \in \mathcal{B}\left(M^{f}\right)$, and therefore

$$
g=\frac{d \tilde{\mu}_{\tilde{\tilde{x}}}^{\eta}}{d \tilde{\lambda}_{\tilde{x}}^{\eta}}
$$

$\tilde{\lambda}_{\tilde{x}}^{\eta}$-almost everywhere on $\eta(\tilde{x})$.

\section{Proof of the Sufficiency for the Entropy Formula}

Assume that $\mu$ has the SRB property. Our purpose in this section is to prove that the identity (1.7) holds. Due to the Margulis-Ruelle inequality, it suffices to prove the inequality

$$
h_{\mu}(f) \geq \int \sum_{i} \lambda^{(i)}(x)^{+} m_{i}(x) d \mu(x),
$$

which is equivalent to

$$
h_{\tilde{\mu}}(\tau) \geq \int \sum_{i} \lambda^{(i)}(\tilde{x})^{+} m^{(i)}(\tilde{x}) d \tilde{\mu}(\tilde{x}),
$$

because of the identity $h_{\tilde{\mu}}(\tau)=h_{\mu}(f)$ and (2) of Proposition 2.1

Let $\eta$ be a partition of $M^{f}$ as introduced in Proposition 3.2 By the general properties of entropies and (1) of Proposition 3.2, one has

$$
h_{\tilde{\mu}}(\tau) \geq h_{\tilde{\mu}}(\tau, \eta)=H_{\tilde{\mu}}\left(\tau^{-1} \eta \mid \eta\right)=-\int \log \tilde{\mu}_{\tilde{x}}^{\eta}\left(\left(\tau^{-1} \eta\right)(\tilde{x})\right) d \tilde{\mu}(\tilde{x}) .
$$

Put $I=\bigcup_{n=1}^{+\infty} I_{n}$ (see the proof of Proposition 3.2) and $J=M^{f} \backslash I$. Note that the restrictions of $\eta$ and $\tau^{-1} \eta$ to $J$ constitute the partition into single points. Hence, for each $\tilde{x} \in J$,

$$
\log \tilde{\mu}_{\tilde{x}}^{\eta}\left(\left(\tau^{-1} \eta\right)(\tilde{x})\right)=0
$$


On the other hand,

$$
\int_{J} \sum_{i} \lambda^{(i)}(\tilde{x})^{+} m^{(i)}(\tilde{x}) d \tilde{\mu}(\tilde{x})=0 .
$$

Therefore, without loss of generality, we may assume that $\tilde{\mu}(I)=1$.

For $\tilde{\mu}$-a.e. $\tilde{x} \in M^{f}$ we may define

$$
\begin{aligned}
X(\tilde{x}) & =\tilde{\mu}_{\tilde{x}}^{\eta}\left(\left(\tau^{-1} \eta\right)(\tilde{x})\right), \\
Y(\tilde{x}) & =\frac{g(\tilde{x})}{g(\tau \tilde{x})}, \\
Z(\tilde{x}) & =\left|\operatorname{det}\left(\left.T_{x_{0}} f\right|_{E^{u}(\tilde{x})}\right)\right| .
\end{aligned}
$$

It is easy to see that $X, Y$ and $Z$ are all measurable and $\tilde{\mu}$-a.e. finite functions on $M^{f}$. We first make the following claims, whose proofs will be given later.

Claim 4.1 $X=Y Z^{-1}, \tilde{\mu}$-a.e.

Claim $4.2 \quad$ (a) $\log Z \in L^{1}\left(M^{f}, \tilde{\mu}\right)$;

(b) $\int \log Z d \tilde{\mu}=\int \sum_{i} \lambda^{(i)}(\tilde{x})^{+} m^{(i)}(\tilde{x}) d \tilde{\mu}(\tilde{x})$.

Claim 4.3 (a) $\log Y \in L^{1}\left(M^{f}, \tilde{\mu}\right)$;

(b) $\int \log Y d \tilde{\mu}=0$.

From these and (4.2) one can immediately obtain (4.1) and complete the proof of the sufficiency.

Proof of Claim 4.1. First notice that for $\tilde{\mu}$-a.e. $\tilde{x},\left.\left(\tau^{-1} \eta\right)\right|_{\eta(\tilde{x})}$ is $\left(\tilde{\mu}_{\tilde{x}}^{\eta}\right.$ - $\left.\operatorname{cod} 0\right)$ a countable partition. Then for $\tilde{\mu}$-a.e. $\tilde{x}$, for any $\tilde{y} \in\left(\tau^{-1} \eta\right)(\tilde{x})$ and a sufficiently small neighborhood of $\tilde{y}$, denoted by $B$, we have

$$
\begin{aligned}
\tilde{\mu}_{\tilde{x}}^{\tau^{-1} \eta}(B) & =\frac{1}{X(\tilde{x})} \tilde{\mu}_{\tilde{x}}^{\eta}\left(\left(\tau^{-1} \eta\right)(\tilde{x}) \cap B\right) \\
& =\frac{1}{X(\tilde{x})} \int_{\left(\tau^{-1} \eta\right)(\tilde{x}) \cap B} g(\tilde{z}) d \tilde{\lambda}_{\tilde{x}}^{\eta}(\tilde{z}) ;
\end{aligned}
$$

on the other hand,

$$
\begin{aligned}
\tilde{\mu}_{\tilde{x}}^{\tau^{-1} \eta}(B) & =\tilde{\mu}_{\tau \tilde{x}}^{\eta}(\tau B) \\
& =\int_{\eta(\tau \tilde{x}) \cap(\tau B)} g(\tilde{z}) d \tilde{\lambda}_{\tau \tilde{x}}^{\eta}(\tilde{z}) \\
& =\int_{\left(\tau^{-1} \eta\right)(\tilde{x}) \cap B} g(\tau \tilde{z})\left|\operatorname{det}\left(\left.T_{z_{0}} f\right|_{E^{u}(\tilde{z})}\right)\right| d \tilde{\lambda}_{\tilde{x}}^{\eta}(\tilde{z})
\end{aligned}
$$

Since $B$ is arbitrarily chosen, we obtain for $\tilde{\mu}$-a.e. $\tilde{x}$

$$
\frac{1}{X(\tilde{x})} g(\tilde{y})=g(\tau \tilde{y})\left|\operatorname{det}\left(\left.T_{y_{0}} f\right|_{E^{u}(\tilde{y})}\right)\right|
$$

for $\tilde{\lambda}_{\tilde{x}}^{\eta}$-a.e. $\tilde{y} \in\left(\tau^{-1} \eta\right)(\tilde{x})$. This implies that for $\tilde{\mu}$-a.e. $\tilde{x}$ one has

$$
X(\tilde{y})=Y(\tilde{y}) Z(\tilde{y})^{-1}, \quad \tilde{\mu}_{\tilde{x}}^{\tau^{-1} \eta} \text {-a.e. } \tilde{y} \in\left(\tau^{-1} \eta\right)(\tilde{x}),
$$

as $X(\tilde{x})=X(\tilde{y})$ for each $\tilde{y} \in\left(\tau^{-1} \eta\right)(\tilde{x})$. Claim 4.1 follows from this. 
Proof of Claim 4.2. By assumption (1.6) and the multiplicative ergodic theorem, it is clear that $\log Z \in L^{1}\left(M^{f}, \tilde{\mu}\right)$ and

$$
\int \log Z d \tilde{\mu}=\int \sum_{i} \lambda^{(i)}(\tilde{x})^{+} m^{(i)}(\tilde{x}) d \tilde{\mu}(\tilde{x}) .
$$

Proof of Claim 4.3. By Claim 4.1,

$$
\log X=\log Y-\log Z \leq 0, \quad \tilde{\mu} \text {-a.e., }
$$

which implies

$$
\log ^{+} Y \leq \log ^{+} Z, \quad \tilde{\mu} \text {-a.e. }
$$

Hence, by Claim $4.2, \log ^{+} Y \in L^{1}\left(M^{f}, \tilde{\mu}\right)$. Then this claim follows from the subadditive ergodic theorem.

Now we have completed the proof of the first part of Theorem 1.1, i.e.. if the invariant measure $\mu$ has the SRB property, the entropy formula (1.7) holds.

\section{NeCessity for the Entropy Formula}

Finally we discuss the remaining part of Theorem 1.1. We indicate that, with some necessary modifications as worked out in Section 3, the necessity part of our main theorem can be proved along the same line given in $\S \S 3-8$ in Chapter VI of Liu and Qian [8]. In order to reduce the length of the paper, we now only go over the main ideas. Detailed proof will be given somewhere else.

We first reduce the problem to its ergodic case. Let $\tau:\left(M^{f}, \tilde{\mu}\right) \rightarrow\left(M^{f}, \tilde{\mu}\right)$ be as introduced above. If $\tilde{\mu}$ is not ergodic, then according to Rokhlin [14, there exists a unique $\left(\tilde{\mu}\right.$-mod 0) measurable partition $\zeta$ of $M^{f}$ fixed under $\tau$ (i.e. $\tau^{-1} C=C$ for each $C \in \zeta)$, such that $\left.\tau\right|_{C}:\left(C, \tilde{\mu}_{C}\right) \rightarrow\left(C, \tilde{\mu}_{C}\right)$ is ergodic for $\tilde{\mu}_{\zeta}$-a.e. $C \in \zeta$, where $\left\{\tilde{\mu}_{C}\right\}_{C \in \zeta}$ is a canonical system of conditional measures of $\tilde{\mu}$ associated with $\zeta$ and $\tilde{\mu}_{\zeta}$ is the measure on the factor space $M / \zeta$ induced by $\tilde{\mu}$.

Assume now that the entropy formula (1.7) holds. Since

$$
h_{\tilde{\mu}}(\tau)=\iint \sum_{i} \lambda^{(i)}(\tilde{x})^{+} m^{(i)}(\tilde{x}) d \tilde{\mu}_{C}(\tilde{x}) d \tilde{\mu}_{\zeta}=\int h_{\tilde{\mu}_{C}}(\tau) d \tilde{\mu}_{\zeta},
$$

and, by the Margulis-Ruelle inequality,

$$
h_{\tilde{\mu}_{C}}(\tau) \leq \int \sum_{i} \lambda^{(i)}(\tilde{x})^{+} m^{(i)}(\tilde{x}) d \tilde{\mu}_{C}(\tilde{x})
$$

for each $C \in \zeta$, one has

$$
h_{\tilde{\mu}_{C}}(\tau)=\int \sum_{i} \lambda^{(i)}(\tilde{x})^{+} m^{(i)}(\tilde{x}) d \tilde{\mu}_{C}(\tilde{x})
$$

for $\tilde{\mu}_{\zeta}$-a.e. $C$. On the other hand, Corollary 3.1 .1 implies that, if $\xi$ is a partition of $M^{f}$ subordinate to $W^{u}$-manifolds of $f$, then $\xi$ refines $\zeta$. Hence, if our main theorem holds in the ergodic case, the arguments above together with the transitivity of conditional measures show that our main theorem holds in general. So we may reduce the theorem to its ergodic case. 
Now assume that $\tau:\left(M^{f}, \tilde{\mu}\right) \rightarrow\left(M^{f}, \tilde{\mu}\right)$ is ergodic and $\lambda^{(i)}, m^{(i)}, 1 \leq i \leq s$, are the Lyapunov exponents and their multiplicities. Then, with necessary modifications as presented in Section 3, techniques in Liu and Qian [8] can be applied to show that

$$
h_{\tilde{\mu}}(\tau)=H_{\tilde{\mu}}(\eta \mid \tau \eta)
$$

if $\eta$ is a partition of the type given in Proposition 3.2. Consequently, by the entropy formula,

$$
H_{\tilde{\mu}}(\eta \mid \tau \eta)=\sum_{i}\left(\lambda^{(i)}\right)^{+} m_{i} .
$$

Now put $J^{u}(\tilde{x})=\left|\operatorname{det}\left(\left.T_{x_{0}} f\right|_{E_{\tilde{x}}^{u}}\right)\right|$ for $\tilde{\mu}$-a.e. $\tilde{x} \in M^{f}$ and define a function by

$$
\rho(\tilde{y})=\frac{\triangle(\tilde{x}, \tilde{y})}{L(\tilde{x})} \quad \text { for } \tilde{y} \in \eta(\tilde{x}),
$$

where

$$
\triangle(\tilde{x}, \tilde{y})=\prod_{k=1}^{+\infty} \frac{J^{u}\left(\tau^{-k} \tilde{x}\right)}{J^{u}\left(\tau^{-k} \tilde{y}\right)}, \quad \forall \tilde{y} \in \eta(\tilde{x}),
$$

and

$$
L(\tilde{x})=\int_{\eta(\tilde{x})} \triangle(\tilde{x}, \tilde{y}) d \tilde{\lambda}_{\tilde{x}}^{\eta}(\tilde{y})
$$

for $\tilde{\mu}$-a.e. $\tilde{x}$. Then it can be shown that (5.2) implies that

$$
d \tilde{\mu}_{\tilde{x}}^{\eta}=\rho d \tilde{\lambda}_{\tilde{x}}^{\eta} \text { for } \tilde{\mu} \text {-a.e. } \tilde{x} .
$$

That is,

$$
\tilde{\mu}_{\tilde{x}}^{\eta} \ll \tilde{\lambda}_{\tilde{x}}^{\eta} \text { for } \tilde{\mu} \text {-a.e. } \tilde{x} \text {. }
$$

From this, it is then easy to obtain that

$$
\pi \tilde{\mu}_{\tilde{x}}^{\eta^{\prime}} \ll \lambda_{\tilde{x}}^{u} \text { for } \tilde{\mu} \text {-a.e. } \tilde{x},
$$

where $\eta^{\prime}$ is the measurable partition subordinate to $W^{u}$-manifolds of $(f, \mu)$ as defined in Proposition 3.2. Consequently, we have

$$
\pi \tilde{\mu}_{\tilde{x}}^{\xi} \ll \lambda_{\tilde{x}}^{u} \text { for } \tilde{\mu} \text {-a.e. } \tilde{x},
$$

for any measurable partition $\xi$ subordinate to $W^{y}$-manifolds of $(f, \mu)$, i.e., $\mu$ has the SRB property.

\section{ACKNOWLEDGEMENT}

The authors wish to thank Professor Peidong Liu for many useful discussions. The paper was finished while S. Z. was visiting the School of Sciences, Purdue University. 


\section{REFERENCES}

[1] J. Bahnmüller and P.-D. Liu, Characterization of measures satisfying Pesin's entropy formula for random dynamical systems, J. Dynam. Differential Equations, 10 (1998), 425-448. MR 99j:58118

[2] H. Y. Hu, Pesin's formula for an expanding endomorphism, Adv. in Math. (China), 19 (1990), 338-349. MR 91i:58080

[3] A. Katok, J. M. Strelcyn, F. Ledrappier, and F. Przytycki, Invariant Manifolds, Entropy and Billiards; Smooth Maps with Singularities, Lecture Notes in Math., Vol. 1222, SpringerVerlag, 1986. MR 88k:58075

[4] F. Ledrappier and J. M. Strelcyn, A proof of the estimation from below in Pesin's entropy formula, Ergod. Th. \& Dynam. Sys., 2 (1982), 203-219. MR 85f:58070

[5] F. Ledrappier and L.-S. Young, The metric entropy of diffeomorphisms, Part I: Characterization of measures satisfying Pein's formula, Ann. of Math., 122 (1985), 509-539. MR 87i:58101a

[6] F. Ledrappier and L.-S. Young, Entropy formula for random transformations, Probab. Th. Rel. Fields, 80 (1988), 217-240. MR 90d:58079

[7] P.-D. Liu, Pesin's entropy formula for endomorphisms, Nagoya Math. J., 150 (1998), 197-209. MR 99h:58108

[8] P.-D. Liu and M. Qian, Smooth Ergodic Theory of Random Dynamical Systems, Lecture Notes in Math., Vol. 1606, Springer-Verlag, 1995. MR 96m:58139

[9] R. Mañé, A proof of Pesin's formula, Ergod. Th.\& Dynam. Sys., 1 (1981), 95-102. MR 83b:58042

[10] R. Mañé, Ergodic Theory and Differentiable Dynamics, Springer-Verlag, 1987. MR 88c:58040

[11] Ya. B. Pesin, Lyapunov characteristic exponents and smooth ergodic theory, Russ. Math. Surveys, 32 (1977), no. 4, 55-114. MR 57:6667

[12] C. Pugh and M. Shub, Ergodic attractors, Trans. Amer. Math. Society, 312 (1989), 1-54. MR 90h:58057

[13] M. Qian and Z.-S. Zhang, Ergodic theory for Axiom A endomorphisms, Ergod. Th. \& Dynam. Sys. 15 (1995) 133-147. MR 96a:58118

[14] V. A. Rokhlin, Lectures on the theory of entropy of transformations with invariant measures, Russ. Math. Surveys, 22 (1967), no. 5, 1-54. MR 36:349

[15] D. Ruelle, Ergodic theory of differentiable dynamical systems, Publ. Math. IHES, 50 (1979), 27-58. MR 81f:58031

[16] D. Ruelle and M. Shub, Stable manifolds for maps, in Global Theory of Dynamical Systems (Z. Nitecki and C. Robinson, Eds.), Lecture Notes in Math., Vol. 819, Spinger-Verlag, 1980, pp. 389-392. MR 82e:58055

[17] P. Thieullen, Fibres dynamiques Entropie et dimension, Ann. Inst. Henri Poicaré, Analyse Non Linéaire, 9 (1992), 119-146. MR 93k:58138

[18] P. Walters, An Introduction to Ergodic Theory, Springer, New York, 1982. MR 84e:28017

[19] Shu Zhu, Unstable manifolds for endomorphisms, Science in China (Series A), 41 (1998), 147-157. MR 99c:58100

School of Mathematical Sciences, Peking University, Beijing 100871, P. R. China

School of Mathematical Sciences, Peking University, Beijing 100871, P. R. China 